\title{
RELACIÓN ENTRE LA CONDUCTA SUICIDA Y EL CONCEPTO DE MUERTE
}

\author{
Augusto Perez Gómez. PhD. \\ Luisa Marina Cortés. Ps. \\ Universidad de Los Andes
}

\begin{abstract}
RESUMEN
Para analizar la relación entre el suicidio y la concepción de muerte, se partió del siguiente supuesto: La concepción que un individuo posea de la muerte puede ser un indicador de riesgo de la intención suicida. Esta investigación estudió las concepciones de muerte predominantes dentro de la población que ha intentado suicidarse y la que sólo presenta ideación, comparándolas con las encontradas en la población normal. La muestra estuvo formada por 90 sujetos, distribuidos en tres grupos (intento, ideación y normal). Se les aplicaron dos instrumentos, la entrevista de diagnóstico psiquiátrico (PDI-R) y el instrumento de concepción de muerte y suicidio construído especificamente para esta investigación. Los datos obtenidos fueron analizados a través de la prueba $\chi^{2}$ y el análisis de varianza (Anova), además se consideraron bajo dos condiciones: Grupo (relacionado con la distribución inicial de las muestras) y Conglomerados (relacionado con la redistribución de los grupos dada por la homogeneidad de las puntuaciones en las variables de los conceptos de muerte). A través del análisis comparativo se llegó a considerar que existe una relación entre la concepción de muerte y la intención suicida de los individuos, de tal forma que se puede considerar el concepto de muerte como un indicador de riesgo suicida.
\end{abstract}

Palabras Claves: Conducta suicida, intención suicida, ideación suicida, concepto de muerte, indicador de riesgo suicida.

\begin{abstract}
In order to analyze the relation between suicide and the conception of death the following construct was held: the conception that an individual might have about death is a potential risk indicator of the suicidal ideation. This research studied the conceptions of death predominant in the population that has attempted suicide and that of the population that only presented suicidal ideation in comparison with the conceptions found in the normal population. The sample was formed by 90 individuals distributed in three experimental groups (intent, ideation and normal). Two instruments were used with the sample: the Psychiatric Diagnostic Interview (PDI-R) and the Conception of Death and Suicide specially developed for this study. The data were analyzed under the $\chi^{2}$ statistic and the ANOVA. Two conditions were considered also for the data analysis: Group, regarding the initial distribution of the subsamples, and Cluster, regarding the redistribution of the groups given by the homogeneity of the scores in the variables related to the concepts of death. The analysis found grounds to confirm a relation between the conception of death and the suicidal intention of the individuals in a way that the conception of death could be considered a risk indicator of suicide.
\end{abstract}

Key words: suicidal behavior, suicidal intent, suicidal ideation, conception of death, suicide risk indicator.

Correspondencia: Dr. Augusto Pérez Gómez en el Programa "La Casa" de la Universidad de los Andes.- Colombia. Cll 33A No 13 - 58 Bogotá - Colombia. E-Mait: Aperez\&cdcnet.uniandes.edu.co 
Para lograr conocer el concepto de muerte de una determinada persona es necesario distinguir qué es lo que ha experimentado como sufrimiento y cómo se ha relacionado esto con su vida, así como qué es lo que para él es importante y lo que teme perder ante la muerte. También las creencias, la religión que se profesa, forman parte de los factores que determinan el modo en que las personas pueden valorar a la muerte, ya sea con un signo negativo, con uno positivo, o simplemente sin ningún valor. La muerte implicaria entonces la anticipación del sufrimiento y la pena del hombre, eventos que han sido desligados de la vida en la medida en que responden a su antitesis, en la medida en que son su negación.

La muerte implica la subjetividad misma, el mundo de lo desconocido. El temor a la muerte no radica en lo que la vida es o fue, radica en el temor de penetrar en la muerte, en ese mundo del cual no se tiene ningún tipo de conocimiento, la posibilidad del no ser, del sufrimiento o del castigo que puede implicar la muerte.

Desde la psicologia muchos autores han tratado de analizar lo que significa la muerte. Para Rojas (1984) existen dos factores singulares en la búsqueda del sentido de la muerte: a) La muerte como acontecimiento de la naturaleza. b) La muerte como algo especifico de la existencia humana.

La primera concepción retoma los postulados de Epicuro. Aqui la muerte no concierne a la existencia humana, sino que expresa y reconoce la idea de un final, que desde el exterior avanza hacia la vida y la detiene. En la segunda, en donde la muerte cobra su verdadero sentido, ésta puede ser entendida de tres formas: como comienzo de un ciclo vital, en donde se retoma la idea de la inmortalidad del alma, la reencarnación y la transmigración; como fin de un ciclo personal, considerada desde la filosofia, pero en donde el hombre concibe la muerte como el fin de la etapa existencial de sí mismo; ó como posibilidad existencial, es decir, "la posible imposibilidad de la existencia" (Rojas, 1984).

Weisman (1982) analizó las 20 actitudes hacia la muerte que Weber postuló, sintetizándolas en cuatro ideas principales:

a) La muerte como ilusión y extensión de la vida: Esta concepción afirma que la muerte es una mentira, una ilusión y el inicio de otra forma de vida. La creencia aqui sobre la inmortalidad personal no hace referencia únicamente a una nueva vida o una continuación de ésta, sino que también se contempla la posibilidad de sobrevivir en forma simbólica a través de los objetos, conocimientos o sentimientos. En esta concepción, la muerte significa más transición que extinción.

b) La muerte como un hecho de vida inevitable e inexorable: Esta concepción puede relacionarse con la confrontación estoica a lo finito. La muerte puede o no ser aceptada, puede existir oposición a ella a través del miedo, los ritos o la negación, pero tarde o temprano la muerte como fin llegará, de tal forma que el hombre debe apropiarse de su vida y vivirla plenamente, para disfrutar aquello que considera que vale la pena. Morir seria el cumplimiento de un contrato realizado en el momento de nacer.

c) La muerte como explicación y expiación de la vida: Esta seria la concepción de los que divinizan la muerte, convirtiéndose ésta en la razón fundamental del estar vivo. Sin embargo, la muerte tiene la capacidad de igualar a todos los seres humanos, siendo por tanto, más real que la propia vida. La muerte desde esta concepción, sería liberación, retribución y recompensa.

d) La muerte como exigencia y derrota de la vida: En esta concepción la muerte implicaría la aniquilación de la vida, es un enemigo, sencillamente una calamidad. Aqui la muerte acaba con lo que dió sentido a la vida y por lo tanto gana en la lucha de la trascendencia.

Hendin (1965) fue el primer autor que trató de ahondar en el problema de la concepción de muerte como tal y su relación con el suicidio. Expone seis tipos fundamentales de concepción de muerte y los fundamenta con casos donde los relaciona con el suicidio. Hendin señala que: "Sus actitudes frente a la muerte, hecho y proceso, y al Más allá, deben ser conocidas de nosotros si es que pretendemos conocer del todo lo que motivó su intento" (1965; p.42).

Los seis tipos fundamentales de muerte que expone Hendin (1965) son los siguientes:

a) La muerte como abandono punitivo: Referida a la sensación de dominio omnipotente por mediación de la muerte. El morir 
implica tener la posibilidad de castigar a un otro por un comportamiento no deseado.

b) La muerte como homicidio a la inversa: Esta concepción es la básica, según Freud (1916) en el suicidio. Para él, no existía suicidio $\sin$ que antes estuviera precedido del deseo reprimido de matar a otra persona, considerada antes como el objeto de amor.

c) La muerte como reunión: Cuando una persona ha tenido una pérdida significativa, ve en el suicidio la posibilidad de reencontrarse en el Más allá con la persona amada que ha perdido. El morir y la muerte, significan para este ser el reencuentro que tanto anhela. Frecuentemente el énfasis no va puesto en la muerte, sino en la satisfacción ulterior; además, no es necesario que la persona perdida haya muerto, sino que en muchos casos se considera que no puede haber reencuentro en vida, sino únicamente en muerte.

d) La muerte como renacer: Se relaciona, con la necesidad de la persona de retroceder y volver a empezar, el deseo de cambiar lo que ya en vida es incambiable y de volverse omnipotente. "Para una persona que sufre fantasías como ésta, la idea de morir tiene un poderoso atractivo" (Hendin; 1965; p.47)

e) La muerte como autocastigo: Ligada a las presiones culturales y religiosas que envuelven la vida cotidiana. Es la mejor forma de expiar las culpas por las acciones realizadas que no van acorde con los mandatos sociales y religiosos. Lo que se considera como un fracaso, genera en el individuo un altisimo odio contra si mismo, y el suicidio equivale a un castigo auto-impuesto por haber fracasado. (Hendin; 1965).

f) El paciente que se ve a si mismo como ya muerto: En palabras de Hendin (1965, p. 50 ), "los sentimientos poderosos de falta de apego, agresividad reprimida y una afectividad marchita son percibidos por muchas personas como el equivalente de la muerte emocional, o del trance de morir emocionalmente".

Vidal \& Alarcón (1986) estudiaron las concepciones de muerte más comunes entre los suicidas y encontraron 9 concepciones de muerte específicas:

1. La muerte como un deseo de evasión, de escapar, de dormir para liberarse del conflicto. 2. La muerte como un deseo de vengan$z a$, de control hostil, de poderio omnipotente sobre el objeto. 3. La muerte como un deseo de castigo, de expiación, de sacrificio propiciado por sentimientos de culpa. 4. La muerte como un deseo apasionado de reunión erótica con un objeto amado. 5. La muerte como un deseo de conmover a otros, de influir sobre sus sentimientos. 6 . La muerte como un deseo de renacer, de comenzar una nueva vida por fantasías de fracaso. 7. La muerte como un deseo de liberación, de paz o de nirvana. 8. La muerte como un deseo de reconquista y de rehabilitación de prestigio, honor y de gloria. 9. La muerte como un deseo de aniquilación, de desintegración del yo.

Se considera que dependiendo del cómo se asuma la muerte, existe la posibilidad de que una persona acceda o no al suicidio. Asimismo, el hombre está rodeado por muchas concepciones tanto de vida como de muerte que influyen en su cotidianeidad. Quizás se entremezclen las concepciones filosóficas, religiosas y psicológicas, pero no debe olvidarse que todas ellas permiten dar al ser hu-mano un sentido a su existencia y a su muerte.

Para analizar la relación entre el suicidio y la concepción de muerte, se partirá del siguiente supuesto: La concepción que un individuo posea de la muerte puede ser un indicador de riesgo de la intención suicida. Esta investigación estudiará las concepciones de muerte comunes dentro de la población que ha intentado suicidarse y la que tiene una fuerte ideación suicida pero que no ha realizado ningún intento; esta información se comparará con las concepciones de muerte de la población general con las características sociodemográficas similares a las de los grupos de estudio. A través del análisis comparativo se pretende llegar a conocer si existe o no una relación entre la concepción de muerte y la intención suicida de los individuos, de tal forma que pueda llegar a considerarse dicha concepción como un indicador de riesgo suicida. Por lo tanto, el objetivo general de esta investigación es brindar a los terapeutas que trabajan con el suicidio, un instrumento para identificar el grado de riesgo de un paciente.

\section{MÉTODO \\ Sujetos}

El estudio contó con la participación de 90 sujetos, 46 hombres y 44 mujeres, todos ellos de nacionalidad colombiana; con edades que 
oscilaban entre los 20 y los 35 años (adultez joven) pertenecientes a los niveles socio-económicos bajo y medio. Con respecto a su nivel educativo oscilaron entre la primaria y la formación universitaria.

La muestra se dividió en tres grupos, tomando en cuenta las caracteristicas específicas de cada uno:

Grupo 1: Formado por 30 sujetos (13 hombres y 17 mujeres) que realizaron al menos un intento de suicidio durante el curso del último año y que no recibieron tratamiento terapéutico a causa del intento. De la misma forma, se tomó en cuenta que no existiera un diagnóstico psiquiátrico de trastorno psicótico con el fin de evitar la influencia de alteraciones de pensamiento $y$ afecto caracteristicos de dicha patología que no permiten ser controlados. Se tomaron en cuenta intentos de suicidio de tipo racional, neurótico y relacionados con trastornos depresivos.

Grupo 2: Formado por 30 sujetos (16 hombres y 14 mujeres) que manifestaron haber tenido ideas de suicidio durante los últimos seis meses, sin haber recurrido a ninguna orientación terapéutica.

Grupo 3: Grupo control. Formado por 30 sujetos ( 17 hombres y 13 mujeres) que no han realizado intentos ni presentan ideación suicida de ningún tipo, siendo considerados como los sujetos de la población general de la muestra.

La muestra fue ubicada en varios sectores de la ciudad. Para el grupo de intento se contó con la colaboración de los pacientes del servicio de urgencias del Hospital Regional Kennedy de Bogotá, quienes se encontraban alli por haber realizado una tentativa de suicidio en las últimas 24 horas. De la misma forma, participaron en este grupo estudiantes universitarios que habian realizado una tentativa suicida en el último año. Para el segundo grupo la muestra se obtuvo de la población en general, siendo escogidos los sujetos que presentaban ideación suicida, ya fuera en la actualidad ó en el transcurso de los últimos seis meses. Las personas evaluadas que no cumplian con los requisitos del grupo uno o el grupo dos fueron seleccionadas para conforma el grupo número 3 de la muestra, siempre y cuando cumplieran con las características de dicho grupo.
Los criterios de selección generales (para la muestra total del estudio) fueron los siguientes:

- Orden de llegada (ya fuera al servicio de Urgencias ó al contacto con la investigadora).

- Pertenencia al rango de edad de 20-35 años (adultez joven).

- Ausencia de trastornos de tipo psicótico durante el último año.

- Consentimiento de los sujetos para participar en el estudio. Si este no era dado no se incluían como sujetos de investigación.

Los criterios adicionales específicos para el grupo de intento fueron:

- Presencia de por lo menos una tentativa suicida en un lapso no mayor a un año.

- Ausencia de tratamiento psicoterapéutico relacionado con el intento.

Los criterios adicionales especificos para el grupo de ideación fueron:

- Presencia de ideación suicida durante los últimos seis meses sin tentativa.

- Ausencia de tratamiento psicoterapéutico relacionado con la ideación.

\section{Instrumentos}

A cada sujeto se le administraron dos pruebas: La Entrevista de Diagnóstico Psiquiátrico (PDI-R; Othmer, Othmer, Penick, Powell \& Read, 1989) y el instrumento de concepto de muerte y suicidio construido para esta investigación.

EI PDI-R es una entrevista estructurada cuyo objetivo fundamental es determinar si una persona está sufriendo, o ha sufrido, un desorden psiquiátrico mayor. Revisa sistemáticamente 17 sindromes básicos y 4 derivados, con preguntas simples para determinar la presencia o ausencia de sintomas especificos y características importantes para realizar el diagnóstico. Los 17 sindromes básicos se dividen en tres categorias: Sindromes Enmascarantes, Sindromes Ordinales y el Sindrome Adicional. Los Enmascarantes son: el sindrome cerebral orgánico, el alcoholismo y el abuso de drogas; los Ordinales son: Depresión, mania, esquizofrenia, personalidad antisocial, desorden de somatización, anorexia nerviosa, bulimia, sindrome de estrés post-traumático, desorden obsesivo-compulsivo, desorden fóbico, desorden de pánico, ansiedad 
generalizada y desorden de adaptación. El síndrome adicional es retardo mental. Si el sujeto no ha reunido los criterios para ninguno de los sindromes anteriores y ha presentado en los últimos dos años sintomas psiquiátricos/conductuales que requirieron asistencia se evalúa el último síndrome: Desorden Psiquiátrico no diagnosticado. Los síndromes derivados son: Policonsumo (Alcoholismo más abuso de drogas), desorden esquizo-afectivo (Esquizofrenia más depresión y manía), desorden maníaco-depresivo (Depresión más manía) y bulimarexia (Bulimia más anorexia). En cada sindrome están divididas las preguntas en cuatro secciones fundamentales: Cardinal, significación social, auxiliar y perfil temporal. Si una sección cardinal se califica como positiva se procede a la sección siguiente. Si esto no ocurre se omiten las secciones posteriores y se pasa al otro sindrome; de esta misma manera se califican las otras secciones, haciéndose necesaria la calificación positiva en la sección precedente para poder avanzar con la siguiente del sindrome. EI PDI-R permite obtener un diagnóstico diferencial cuando el paciente posee más de dos sindromes y permite realizar un diagnóstico actual (transeccional) y un diagnóstico de vida (longitudinal).

Merece la pena aclarar que se utilizó la versión experimental del PDI-R, traducción realizada por S. Jiménez del Programa "La Casa", para descartar trastornos de tipo psicótico en la muestra, al igual que para conocer si los sujetos han presentado (o presentan en el momento de la aplicación) ideación suicida, considerando únicamente la presencia más no la frecuencia ni la intensidad. Se utilizaron sólo dos secciones del PDI-R, la de esquizofrenia y la de depresión, la cual fue la que permitió conocer la existencia de la ideación suicida. Si ésta existia (o habia existido) se le preguntaba a los sujetos sobre las características de la misma y el motivo que la habia suscitado, buscando con esta información profundizar en la ideación. Igualmente se profundizó en las caracteristicas del intento (en los sujetos del grupo 1), para tener un contexto más claro sobre el mismo. Su tiempo de aplicación fue de 50 minutos.

En una etapa preparatoria se elaboraron dos instrumentos, uno con el fin de evaluar concepción de muerte y otro cuyo objetivo era evaluar concepción de muerte y suicidio. A estos instrumentos se les realizó un pilotaje con 60 sujetos (seleccionados de la población normal), del cual se concluyó la validez y la confiabilidad (estadistica) del segundo instrumento, rechazando el primero. De este último quedaron sólo 2 items, los cuales se anexaron al instrumento 2. Después de esto, y con las modificaciones al instrumento se realizó una nueva validación, esta vez con jueces (cinco profesionales del área de la salud que han tenido en su trayectoria contacto con pacientes suicidas) la cual arrojó las modificaciones necesarias para aplicar el instrumento a la muestra del estudio.

El instrumento final de concepto de muerte y suicidio ${ }^{\prime}$ (construido para este estudio), se utilizó para analizar la relación entre el concepto de muerte y la posibilidad de que una persona intente o no suicidarse, estando en la capacidad de discriminar entre las concepciones de muerte de la población normal y la población suicida. Este instrumento consta de 10 items, donde los distractores son calificados por los sujetos de 1 a 5 , donde uno significaba total desacuerdo con la frase; dos, desacuerdo; tres, indiferencia; cuatro, acuerdo y cinco total acuerdo; este tipo de calificación se utilizó para los 7 primeros items. Para el 8 y 10 los sujetos debian darle una jerarquia a los distractores donde 1 era la situación en la cual optarian por el suicidio y 8 la situación en la cual no pensarian en esa opción. En el item 9 los sujetos debian calificar los distrac-tores con falso y verdadero de acuerdo a su propio juicio. El instrumento analiza fundamentalmente los conceptos de muerte a través de las respuestas dadas en los items del 5 al 7, lo que muestra el concepto general que posee el sujeto. Cada concepto de muerte tenía dos o tres distractores en toda la prueba, lo cual permitia observar la constancia del concepto del sujeto. De la misma forma, este instrumento brinda la posibilidad de analizar otro tipo de indicadores de riesgo como son: Valoración del suicidio, contexto suicida, motivación del deseo de muerte, deseos de muerte relacionados, función de la muerte, factores precipitantes y fantasias suicidas asociadas. Su tiempo de aplicación fue de 20 minutos aproximadamente.

La aplicación de las dos pruebas se realizó de manera individual. 


\section{Procedimiento}

Después de validarse el instrumento de concepto de muerte y suicidio, se procedió a realizar los contactos para conseguir la muestra del estudio, obteniéndose una respuesta positiva por parte de la Unidad de Salud Mental del Hospital Regional Kennedy. De igual forma, se procedió a contactar las personas de la Universidad que por el conocimiento de su historia presentaron o presentaban intentos 0 ideaciones suicidas. A través del contacto con ellos, la población a la cual se podía acceder aumentó en la medida en que, al parecer y tal como se demostró, la conducta suicida (ya sea intento o ideación) se conforma como un elemento de vinculación entre las personas. Igualmente se tuvo acceso a poblaciones de estratos sociales más bajos, a través de personas "contacto" que sabian el objeto del estudio y que conociendo las caracteristicas de los individuos los pusieron en comunicación directamente con las investigadoras. Lo mismo ocurrió con los sujetos del grupo normal. Posteriormente, se concertó una cita con estas personas (excepto las que se encontraban en el servicio de urgencias del Hospital Regional Kennedy) ya fuera en sus hogares ó en su sitio de trabajo para hablar sobre la investigación.

Después del primer contacto, se procedió con una entrevista informal donde se buscaba obtener información con respecto a la problemática de estudio, para así poder clasificar a los sujetos dentro de alguno de los tres grupos de investigación.

El paso siguiente consistió en la aplicación de dos secciones del PDI-R. En primera instancia la de Esquizofrenia, que era la más importante para descartar trastornos de tipo psicótico; cuando estos eran descartados se procedió a aplicar la segunda sección, la de Depresión, la cual podía ahondar en ciertas caracteristicas asociadas al suicidio al igual que profundizar en ciertas caracteristicas especificas de la ideación. Posteriormente, se procedió a la aplicación del instrumento. Se leyeron con ellos las instrucciones impresas y se les comunicó la posibilidad de realizar alguna pregunta si no les era claro algún aspecto del mismo. Esto ocurrió con la mayoría de los sujetos, es decir, que cada uno diligenciara su propio instrumento. No sucedió así con los sujetos del servicio de urgencias, con los cuales se vió la necesidad de leerles el cuestionario, distractor por distractor y anotar su respuesta frente a cada uno.

Los análisis estadísticos se realizaron con el programa Statistical Package for the Social Science (SPSS), versión 3.

\section{Análisis Estadísticos}

Los resultados arrojados por el instrumento fueron analizados estadisticamente con tres pruebas: Chi-Cuadrado $\left(\chi^{2}\right)$, Análisis de Varianza $(F)$ paramétrico y Análisis de Conglomerados (Cluster Analysis). Se empleo la prueba $\chi^{2}$ para determinar diferencias en la distribución de proporciones en las respuestas dadas en cada uno de las distractores de todos los items; en cuanto al análisis de varianza se utilizó para determinar diferencias de promedios en las puntuaciones totales en las sub-escalas de la prueba de conceptos de muerte.

El análisis de conglomerados pretendió cumplir un fin específico: definir un perfil en función del concepto de muerte. Si bien es posible que se encontraran diferencias de promedios en cada una de las sub-escalas de concepto de muerte, es relevante conocer un perfil constituido por todos los conceptos de muerte de manera integrada. Tal integración no se hace simplemente seleccionando en cada grupo los promedios más altos en cada sub-escala; se hace imperativo que exista homogeneidad en las respuestas de los sujetos.

Estos análisis se aplicaron a las variables (cada uno de los distractores) en su forma absoluta y en su forma relativa. Los análisis estadísticos realizados a las variables en su forma absoluta implican el promedio de datos por muestra sobre el número total muestral $(\mathrm{N}=90)$. Por su parte, los datos de las variables en su forma relativa son el resultado de la sumatoria de los puntajes obtenidos en una concepción de muerte (las cuales agrupaban por lo menos 3 variables) sobre la sumatoria de los puntajes obtenidos en todas las concepciones. 
Tabla 1.- Conformación de los Conglomerados

\begin{tabular}{|lccccccccc|}
\hline & \multicolumn{4}{c}{ Muestra a la que Pertenece } & \multicolumn{3}{c|}{ Total } \\
Conglomerados & \multicolumn{4}{c}{ Intento S. } & \multicolumn{2}{c|}{ Ideación S. } & \multicolumn{2}{c|}{ Normal } \\
& $\mathrm{N}$ & $\%$ & $\mathrm{~N}$ & $\%$ & $\mathrm{~N}$ & $\%$ & $\mathrm{~N}$ & $\%$ \\
\hline Conglomerado I & 5 & 16.7 & 9 & 30.0 & 21 & 70.0 & 35 & 38.9 \\
Conglomerado II & 11 & 36.7 & 15 & 50.0 & 9 & 30.0 & 35 & 38.9 \\
Conglomerado III & 14 & 46.7 & 6 & 20.0 & & & 20 & 22.2 \\
\hline
\end{tabular}

De la misma manera, se analizaron de dos formas: la condición grupo, compuesta por intento de suicidio, ideación suicida y normal; y la condición conglomerado (clusters analysis) $\chi_{(4,90)}^{2}=6.66 p<.00001$, en la cual tomando como fundamentales los resultados en los tipos de concepción de muerte se reagruparon los datos de las muestras buscando formar grupos homogéneos en dichas variables. $\mathrm{El}$ resultado de este conglomerado de datos indicaria que el primero (l) sería el que agruparía a la población normal de la muestra, debido a que la mayor parte de este conglomerado está conformado por sujetos de dicho grupo; el conglomerado II agruparia principalmente a la población de ideación suicida y, el conglomerado III agruparia a la población de intento de suicidio como se observa en la Tabla 1.

\section{RESULTADOS}

Para comenzar, es importante anotar que las características demográficas de los grupos también estuvieron sujetas a análisis estadísticos debido a que estas variables no fueron controladas. Se encontró que sólo se presentan diferencias significativas para el "nivel socioeconómico" bajo las dos condiciones de análisis (grupos y conglomerados). Para la condición grupos $\chi_{(6.90\}}^{2}=24.21 \mathrm{p}<.0005$, el $40 \%$ del grupo de intento está conformado por sujetos con una formación básica secundaria, en contraposición al grupo normal, en el cual el $90 \%$ del grupo presenta una formación superior (Técnica o universitaria). Por su parte, el $83.4 \%$ del grupo de ideación se configura con sujetos con una formación superior. Para la condición conglomerados $\chi_{(6,90)}^{2}=$
$17.37 p<.0080$, los tres conglomerados tienen sujetos en los cuatro niveles, aún cuando la distribución varia. Así en el conglomerado I (conformado en su mayoría por el grupo normal) hay un mayor porcentaje de sujetos con formación básica secundaria $(20 \%)$ en comparación con el mismo nivel educativo en el grupo normal (10\%); en cuanto al nivel superior (técnico o universitario) el porcentaje disminuye (77\%). En el conglomerado II (conformado por el grupo de ideación principalmente) no hay mayor variación en la distribución por niveles, notándose únicamente un aumento en el porcentaje de sujetos con formación básica secundaria (20\%). Por su parte, en el conglomerado III (compuesto en su mayoria por sujetos del grupo de intento) existe una disminución en el porcentaje de sujetos con formación básica secundaria $(20 \%)$, manteniendo porcentajes similares a los del grupo de intento en los niveles restantes. Las otras características demográficas como son sexo, nivel socioeconómico y edad no presentaron diferencias significativas.

$\mathrm{El}$ instrumento construido para esta investigación permite analizar los resultados obtenidos de dos maneras: La primera, en la cual se agrupan los hallazgos relacionados con cada pregunta a través de categorias teóricas y la segunda, en la cual se asocian tres preguntas (con sus respectivas variables) de las cuales se obtiene el concepto de muerte determinante para cada sujeto y por lo tanto, para cada muestra.

A continuación se hará la exposición de los resultados relacionados con los conceptos de muerte determinantes entre las muestras. 
Tabla 2.-Concepciones de Muerte con valores relativos bajo la condición conglomerados

\begin{tabular}{|c|c|c|c|c|c|c|c|c|}
\hline \multirow[t]{2}{*}{ Conceptos de Muerte } & \multicolumn{2}{|c|}{ I $(N=35)$} & \multicolumn{2}{|c|}{$\begin{array}{l}\text { Conglomerados } \\
\text { II }(N=35)\end{array}$} & \multicolumn{2}{|c|}{ III $(N=20)$} & \multicolumn{2}{|c|}{ Total } \\
\hline & $M$ & SD & $M$ & SD & $M$ & $\mathrm{SD}$ & $M$ & SD \\
\hline llusión y Extensión ${ }^{a}$ & .09 & .03 & .09 & .03 & .05 & .02 & .08 & .03 \\
\hline Hecho de Vida Inex. & .11 & .05 & .07 & .04 & .07 & .02 & .08 & .04 \\
\hline Explicación y Expiación & .07 & .03 & .07 & .03 & .07 & .02 & .07 & .03 \\
\hline Exigencia y Derrota ${ }^{c}$ & .07 & .03 & .04 & .02 & .06 & .02 & .06 & .03 \\
\hline Abandono Punitivo ${ }^{d}$ & .07 & .03 & .06 & .03 & .07 & .01 & .07 & .03 \\
\hline Homicidio a la Inversa & .08 & .03 & .08 & .03 & .08 & .02 & .08 & .03 \\
\hline Muerte como Reunión & .07 & .04 & .07 & .04 & .06 & .03 & .07 & .03 \\
\hline Muerte como Renacere & .07 & .03 & .13 & .03 & .09 & .02 & .10 & .04 \\
\hline Muerte como Autocastigo' & .06 & .02 & .04 & .02 & .09 & .03 & .06 & .03 \\
\hline $\begin{array}{l}\text { Deseo de Conmover } \\
\text { Deseo de Liberación }\end{array}$ & .09 & .03 & .06 & .03 & .05 & .02 & .07 & .03 \\
\hline \multirow{2}{*}{$\begin{array}{l}\text { Deseo de Liberación }{ }^{9} \\
\text { Deseo de Reconquistah }\end{array}$} & .09 & 05 & .14 & .04 & .11 & .01 & .11 & .04 \\
\hline & .06 & .03 & .04 & .02 & .09 & .03 & .06 & .03 \\
\hline Visto como ya muerto' & .07 & .03 & .10 & .04 & .10 & .01 & .09 & .03 \\
\hline $\begin{array}{ll}\mathrm{a}^{\mathrm{a}} F_{(2,89)}=17.33, & p<.0 \\
{ }^{\mathrm{b}} F_{(2,89)}=11.94, & p<.0 \\
{ }^{\mathrm{c}} F_{(2,89)}=9.54, & p<.0\end{array}$ & & 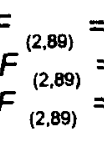 & $\begin{array}{r}3.86 \\
39.18 \\
35.01\end{array}$ & $\begin{array}{l}p<.0246 \\
p<.0000 \\
p<.0000\end{array}$ & & $\begin{array}{l}F_{(2,89)} \\
F_{(2,89)} \\
F_{(2,89)}\end{array}$ & $\begin{array}{l}=11.12 \\
=29.07 \\
=8.72\end{array}$ & $\begin{array}{l}p<.0000 \\
p<.0000 \\
p<.0004\end{array}$ \\
\hline
\end{tabular}

Concepción de muerte relacionada con el grupo normal (Conglomerado I)

Se encontraron los siguientes conceptos predominantes en el grupo normal: "Muerte como ilusión y extensión de la vida", contraste que se establece entre conglomerado I y conglomerado III como se aprecia en la tabla 2; "Muerte como hecho de vida inexplicable e inexorable" y "Muerte como exigencia y derrota de la vida", contraste que se establece tanto con conglomerado II como con conglomerado III. El concepto "Muerte como abandono punitivo" se observa contraste entre conglomerado I y conglomerado II.

Concepción de Muerte Relacionada con el Grupo de Intento (Conglomerado III)

Considerando los puntajes relativos que se aprecian en la tabla 2, el conglomerado III contrasta con los otros dos en los siguientes conceptos: "Muerte como autocastigo" y "Muerte como reconquista". Con el conglomerado I en "Muerte como renacer" y en "Visto como ya muerto". Con el conglomerado II en "Muerte como exigencia y derrota de la vida".

Concepción de Muerte Relacionada con el Grupo de ldeación (Conglomerado II)

Se encontraron los siguientes contrastes significativos, considerando los puntajes relati- vos: en "Muerte como renacer" y "Muerte como liberación" con respecto a los otros dos conglomerados. En el concepto "Visto como ya muerto" se encontró contraste con el conglomerado I, tal y como se aprecia en la tabla 2.

Concepción de Muente Relacionada con el Nivel Educativo

Los niveles superiores (Técnico y universitario) presentan una clara tendencia a tener como concepto "La muerte como ilusión y extensión de la vida" tanto bajo los valores absolutos $F_{(3.86)}=2.71, p<.049$ como bajo los valores relativos $F_{(3.86)}=3.69, p<.0149$, diferenciándose significativamente de los otros dos niveles educativos (primaria y bachillerato).

Con relación a los conceptos de muerte asociados a los grupos suicidas la variable "nivel educativo" influye en los conceptos de "muerte como reunión" $F_{(3,86)}=3.20, p<.0270$ $y$ "muerte como renacer" $F{ }_{(3,86)}=3.97, p<$ .0105 , donde los sujetos con formación básica primaria tienden a sustentar estos conceptos más que quienes tienen una formación superior (Técnica o universitaria); por otro lado, para el concepto "visto como ya muerto" $F_{(3,86)}=3.27, p<.0249$, los sujetos con un 
nivel de formación básica primaria y los que tienen una formación universitaria presentan mayor preferencia por éste en comparación con quienes tienen una formación técnica.

\section{DISCUSIÓN}

Es posible aceptar la hipótesis básica de esta investigación, donde se considera que sí existe relación entre el concepto de muerte y la conducta suicida, al encontrar diferencias significativas en los conceptos de muerte que teóricamente han sido considerados especificos del suicidio. Concepciones de muerte en donde el paciente se ve a sí mismo como ya muerto y la muerte como renacer, se conforman como los elementos básicos del concepto de muerte en el suicidio, relacionándose como potencializadores del acto suicida. De la misma manera, concepciones como homicidio a la inversa, muerte como liberación y muerte como reunión posibilitan que se geste en el individuo la necesidad de optar por el suicidio como la salida válida y viable de la situación vital en que se encuentran.

Tomar los conceptos de muerte como indicadores permite asumir diferentes niveles de riesgo suicida, ya sea para los individuos que presentan en su historia premórbida un intento o para los que manifiestan solamente ideación. De la misma forma, los conceptos permitirian asumir diferentes tipos de intencionalidad y por consiguiente, diferentes factores desencadenantes de un acto suicida. De esta manera, conceptos de muerte como: "Muerte como renacer", "visto como ya muerto", "muerte como reunión" y "muerte como liberación" son indicadores de alto riesgo suicida en cuanto se basan en crisis existenciales engendrando así suicidios de tipo balance ó intentos de suicidio pasionales (Deshaies, 1949), los cuales serian a su vez predictores de una posible "nueva tentativa" que sí llevaría a la muerte.

Por otro lado, conceptos como "muerte como autocastigo", "muerte como abandono punitivo" y "muerte como reconquista y rehabilitación del prestigio el honor y la gloria" son indicadores de alto riesgo para actos suicidas de tipo cortocircuito o para intentos de suicidio emotivos de acuerdo a la clasificación de Deshaies (1949), los cuales no serian indicadores de riesgo para una nueva tentativa de-bido a que son actos eminentemente impulsivos (acting-out). Esta caracteristica conlleva varias "ventajas", si se toma en cuenta que en el individuo que comete un intento por impulso se generan ciertos sentimientos negativos (ya sea rechazo o culpa) que los hacen desechar la idea del suicidio de sus opciones válidas para solucionar dificultades vitales. Igualmente, la "ideación suicida" en este tipo de intentos, podría manejarse a través de un proceso terapéutico al cual los sujetos acceden mucho más fácilmente que quienes sustentan intentos pasionales o racionales (generados por crisis existenciales).

Un riesgo medio de suicidio estaria relacionado con conceptos de muerte pertenecientes a la población normal y que en este estudio, fueron significativos para la población suicida. Estos conceptos son: "Muerte como hecho de vida inexplicable e inexorable" y "muerte como exigencia y derrota de la vida", los cuales pueden tomarse como la base desde la "normalidad" para establecer la conducta suicida en nuestra población. Aunque no existiera una ideación suicida clara en un individuo, si este posee estos conceptos podría pensarse en la posibilidad de que en él se generará en algún momento de la vida una ideación suicida.

En los conceptos de "muerte como ilusión y extensión de la vida", "muerte como explicación y expiación" y "muerte como un deseo de conmover a otros e influir sobre sus sentimientos" no existiria virtualmente ninguna posibilidad de riesgo suicida, ya que según los resultados arrojados por ésta investigación, los elementos que componen estos conceptos no son generadores de conducta suicida. Con respecto al instrumento construído para esta investigación, se observó su capacidad para discriminar, en la población bogotana especificamente, entre los tres grupos de estudio. Este hecho permite utilizar los resultados arrojados como base para una posible interpretación de la aplicación de dicha prueba. Es importante aclarar que aunque no se obtuvieron diferencias significativas dentro de esta primera aplicación formal del instrumento para todos los distractores, las puntuaciones altas (de 4 o 5) en cualquiera de los distractores pertenecientes a las preguntas calificables de uno a cinco son un indicador relevante hacia la conducta suicida. 
Con relación a los grupos de variables que conformaban los conceptos de muerte también se encontraron diferencias significativas que discriminan entre los tres grupos, esperando por lo tanto encontrar puntuaciones comparables en aplicaciones posteriores del instrumento. Con respecto a los conceptos de muerte considerados para la población normal, en "muerte como hecho de vida inexplicable e inexorable" y en "muerte como exigencia y derrota de la vida" puntuaciones iguales ó superiores a tres se relacionan con la presencia de intención suicida. Para los conceptos de muerte asociados a la población suicida, la muerte como "abandono punitivo", "homicidio a la inversa", "reunión" y "autocastigo" con puntuaciones mayores a tres indicarian presencia de conducta inclinada hacia la intención suicida. Por otro lado, en el concepto de muerte "visto como ya muerto", puntuaciones mayores de 3 implicarian tendencias suicidas hacia ideación, pero si dicha puntuación es superior a 4 se relaciona ya con una intención suicida mucho más clara y fortalecida. En cuanto a los conceptos de muerte como "renacer", "liberación" y "reconquista", puntuaciones mayores a tres se relacionan con la presencia de conducta suicida (intención o ideación). El análisis aquí se relacionaría con el riesgo ya mencionado para cada tipo de concepto de muerte.

\section{LIMITACIONES AL ESTUDIO}

Las limitaciones de la presente investigación se relacionan principalmente con características de la muestra. En primera instancia, hubiera sido más apropiado acercarse al concepto de muerte de la población que ya ha consumado el acto, debido a que este concepto presenta una connotación mucho más exacta para asumir la concepción de muerte como indicador de riesgo. Sin embargo, este acceso, que sólo puede realizarse a través de las personas más cercanas al suicida, puede deformar la real concepción de muerte de la persona que ha acabado con su vida. En vista de que dicho acceso no se utilizó, se opto por contactar a la población suicida que aún no habia consumado el acto, la cual podía dar un acercamiento válido al concepto de muerte en relación con el suicidio.
En segunda instancia, el hecho de que algunos de los sujetos del grupo de intento, presentaran tentativas de tipo impulsivo pudo (en el análisis de grupos principalmente) ocasionar que los resultados no fueran tan contundentes como se esperaba. Sin embargo, esto pudo corregirse y lograr una mayor validez al hacer el análisis de conglomerados, ya que los grupos de estudio se reagruparon por la homogeneidad en los conceptos de muerte en los cuales era mucho más marcada la característica propia de cada muestra. Asi, se llegó a una selección mucho más consistente de las muestras y de los resultados a analizar.

En tercera instancia, en esta investigación no se controlaron las variables demográficas, las cuales pueden influir en los resultados. Aún cuando después de los análisis estadisticos no se presentaron resultados contradictorios con los ya analizados, vale la pena reestructurar la metodologia en un estudio posterior para confirmar los hallazgos encontrados en la presente investigación.

\section{IMPLICACIONES}

El presente estudio tiene varias implicaciones. La primera se relaciona con la posibilidad de incluir el concepto de muerte como un factor indicador del nivel de riesgo suicida. Como pudo demostrarse aqui, algunas concepciones de muerte conllevan cierta tendencia suicida que merece ser tenida en cuenta por las personas que trabajan con el suicidio a nivel individual. De la misma forma, el concepto de muerte, al ser identificado, permite realizar un trabajo preventivo con poblaciones que podrian estar engendrando con el concepto vias de solución eminentemente suicidas.

La segunda, es la posibilidad de profundizar en la relación existente entre el concepto de muerte $y$ el suicidio, llegando a clarificar si el concepto inhibe o posibilita la conducta suicida y conocer hasta qué punto se relacionan e interactúan para llegar a la consumación del acto.

La tercera, se relaciona con el instrumento construido, el cual demostró su capacidad para discriminar la población suicida a través de los conceptos de muerte. Sin embargo, vale la pena resaltar que este instrumento analiza no solamente la concepción de muerte sino, 
además, otros factores circundantes a ésta que se relacionan estrechamente con el suicidio, tales como la valoración, el contexto en el cual se engendra más fácilmente la idea de muerte, la motivación existente detrás del deseo de morir, la función de la muerte, el deseo de muerte, los factores precipitantes asociados y las fantasias suicidas. Este instrumento en su totalidad se constituye en una herramienta apropiada para obtener un conocimiento profundo del contexto psicológico en el que se encuentra inmersa una persona que podria acceder, en un momento u otro de su vida, al suicidio.

Por último, merece la pena emplear este instrumento en otras poblaciones de América Latina con el fin de profundizar en los conceptos que, en cada dinámica cultural particular, tendrian repercusiones en la intencionalidad suicida.

Como conclusión de esta investigación puede considerarse que si se toma en cuenta el concepto de muerte como indicador del riesgo suicida, la evaluación del paciente puede ser mucho más completa debido a que los elementos integrales del concepto permiten que a partir de uno sólo de ellos se pueda inferir la forma general como se concibe la muerte y lo que se busca conseguir a través del suicidio.

\section{REFERENCIAS BIBLIOGRÁFICAS}

Deshaies, G. (1949). La psychologie du suicide. Paris: Presses Universitaires de France.

Freud, S. (1988). Aflicción y Melancolia. Madrid: Alianza (Trabajo original publicado en 1.916). Hendin, H. (1965). El suicidio en Escandinavia: Estudio psicoanalítico de la cultura y el carácter. Barcelona: Ariel.

Othmer, E., Othmer, S., Penick, E.,Powell, B. \& Read, M. (1989). Entrevista de diagnóstico psiquiátrico-revisado (PDI-R). (S. Jimenez, Trad.) Western Psychological Services.

Rojas, E. (1984). Estudios sobre el suicidio. Madrid: Salvat.

Vidal, G. \& Alarcón, R. (1986). Psiquiatria. Buenos Aires: Panamericana.

Weisman, A.D. (1972). On dying and denying: A psychiatric study of terminality. New York: Behavioral publications.

\section{NOTAS}

1 Para mayor información sobre este instrumento puede dirigirse al Dr. Augusto Pérez Gómez en el Programa "La Casa" de la Universidad de los Andes. 